\title{
„Gefühl“ - „Empfindung“- „Affekt-Motorik“
}

\author{
Sabine Trautmann-Voigt
}

$\mathrm{N}$ ach Damasio $(1994,354 \mathrm{f})$ wird unter Gefühl (engl. „emotion“, Synonym für Emotion, Affekt) eine Reihe von Veränderungen verstanden, die im Gehirn und im Körper stattfinden und durch einen bestimmten geistigen Inhalt ausgelöst werden. Gefühle wie Freude, Angst, Wut etc. haben außerdem mit bewussten Wahrnehmungen bzw. mit dem subjektiven Erleben einer Bedeutung in einer Situation zu tun (Krause 2006, 30).

Unter Empfindung (engl. „feeling“, Wahrnehmung von Körperstruktur und Körperzustand) wird eine lokalisierbare Wahrnehmung einer solchen Veränderung, die im Körper stattfindet und dort z.B. als Spannung oder Zittern oder Kälte / Hitze etc. wahrgenommen werden kann, verstanden. „Im großen und ganzen sind Empfindungen ein momentaner Blick auf einen Teil dieser Körperlandschaft.“ (Damasio 1994, 15)

Unter Affekt-Motorik, auch Körper-Fühlen oder „Fühl-Körper“ wird die Mobilisierung dreier körperlicher Systeme verstanden. Die Affekt-Motorik mobilisiert

1. die Expression bzw. den unwillkürlichen Ausdruck von innerem Erleben,

2. die Willkürmotorik, die funktional und zielgerichtet ist,

3. physiologische Reaktionsmuster, die sichtbare motorische Begleitphänomene zu Gefühlen darstellen.
Es ist äußerlich nicht immer sofort differenzierbar, in welcher Weise diese drei körperlichen Systeme bei der Affekt-Motorik zusammenspielen! Sie können ohne Beteiligung des zentralen Nervensystems im limbischen System ablaufen, d.h. die Beteiligung „höherer“ kognitiver Funktionen ist für ihre Auslösung nicht immer notwendig; diese kann aber vorhanden sein (Krause 2006, 29). Bei der Bewegungsanalyse, wie sie hier verstanden wird, spielen diese drei körperlichen Systeme immer zusammen.

Es ist nicht davon auszugehen, dass Affekt-Motorik im Verhältnis 1:1 inneres Erleben nach außen bringt. Denn inneres Erleben kann unwillkürlich oder willkürlich gesteuert sein. Es ist vielmehr neben einer präzisen Beobachtung motorischer Phänomene immer auch eine reflektierte Einschätzung bzw. interpretierende Einordnung in einen komplexen $\mathrm{Zu}$ sammenhang notwendig. Diese Einschätzung erfolgt auf der Basis eines psychologischen Paradigmas, hier der Psychodynamischen Psychotherapie.

Es gibt primäre, d.h. angeborene Gefühle, die auf eben diesen Schaltkreisen des limbischen Systems beruhen, wobei besonders die Amygdalla und der Gyrus cinguli eine wichtige Rolle spielen. Wir sind „zu einer präorganisierten Gefühlsreaktion verdrahtet [...], wenn wir bestimmte Reizmerkmale in der Welt oder im Körper - allein oder kombiniert - wahrnehmen.“ (Damasio 1994, 183) 
Diese primären Gefühle bilden den Grundlagenapparat für die sekundären, d.h. für diejenigen Gefühle, die kategorial benennbar sind, wie z.B. Scham, Ekel, Lust, Müdigkeit, Ärger, Trauer usw. „Gefühle sind also mentale Sensoren für den inneren Zustand des Organismus [...] Freude, Traurigkeit und andere Gefühle sind im Wesentlichen Vorstellungen vom Körper und seinen Bestrebungen, einen Zustand optimalen Überlebens zu erreichen.“ (Damasio 2003, 164)

Wir alle sind in der Lage, körperliche Signale anderer Menschen intuitiv als Gesamteindruck wahrzunehmen. Diese angeborene biologische Fähigkeit, die auf die Spiegelneurone (Rizzolatti et. al. 2001) zurückgeht, ermöglicht schnelles Reagieren und umgeht die weitaus langsameren, bewussten Denkvorgänge. Diese biologische Fähigkeit ist noch keine Empathie im Sinne der Einfühlung. Einfühlung verstehe ich mit Krause $(2006,30)$ als ein bewusstes Erleben und Zuordnen von gefühlsmäßigen Geschehnissen zu einem anderen Menschen. Die kognitiv-emotionalen Voraussetzungen zur Empathie erwirbt ein Kind aber frühestens mit $21 / 2$ Jahren. Solche Prozesse setzen nämlich voraus, den anderen als unabhängig von der eigenen gefühlten Innenwelt wahrnehmen zu können, und finden sich heute z. B. im Konzept der „theory of mind“ bzw. im Konzept der Mentalisierung (Fonagy et. al. 2002).

\section{Literatur}

Damasio, A. (1994): Descartes' Error: Emotion, Reason and the Human Brain. Avon Books, New York

Damasio, A. (2003): Der Spinoza-Effekt. Wie Gefühle unser Leben bestimmen. List, München

Fonagy, P., Target, M. (2002): Neubewertung der Entwicklung der Affektregulation vor dem Hintergrund von Winnicotts Konzept des falschen Selbst. Psyche (56), 839-862

Krause, R. (2006): Emotionen, Gefühle, Affekte. Ihre Bedeutung für die seelische Regulierung. In: Remmel, A., Kernberg, O. F., Vollmoeller, W., Strauß, B. (Hrsg.): Handbuch Körper und Persönlichkeit. Entwicklungspsychologie, Neurobiologie und Therapie von Persönlichkeitsstörungen. Schattauer, Stuttgart

Rizzolatti, G., Fogassi, I., Gallese, V. (2001): Neurophysiological mechanisms underlying the understanding and imitation of action. Nature Rev Neurosci (2), 661-670

Trautmann-Voigt, S., Voigt, B. (2012): Grammatik der Körpersprache. Ein integratives Lehr- und Arbeitsbuch zum Embodiment. 2. Aufl. Schattauer, Stuttgart

\section{Die Autorin}

\section{Dr. phil. Sabine Trautmann-Voigt}

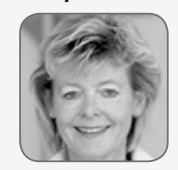

Psychologische und Kinder- und Jugendlichen-Psychotherapeutin in eigener Praxis in Bonn, Tanztherapeutin (ADTR), Leitung des DITAT und der Köln-Bonner Akademie für Psychotherapie (KBAP).
Dr. Sabine Trautmann-Voigt MVZ-Psyche

Bertha-von-Suttner-Platz 6 | D-53111 Bonn info@mvzpsyche.de 